\title{
Matrix metalloproteinase-1 promotes prostate tumor growth and metastasis
}

\author{
SAI MURALI KRISHNA PULUKURI ${ }^{1}$ and JASTI S. RAO ${ }^{1,2}$ \\ Departments of ${ }^{1}$ Cancer Biology and Pharmacology, and ${ }^{2}$ Neurosurgery, \\ University of Illinois College of Medicine at Peoria, Peoria, IL 61656, USA
}

Received November 16, 2007; Accepted December 28, 2007

\begin{abstract}
Cell migration and invasion are critical events during the progression to metastasis. Matrix metalloproteinase-1 (MMP-1) is involved in the progression of human malignancies, but the precise role of MMP-1 in tumor invasion and metastasis remains unclear. In the present study, we investigated the role of MMP-1 in tumor cell invasion and metastasis by overexpressing MMP-1 in prostate cancer cells. Overexpression of MMP-1 in prostate cancer cells increases cell invasion and migration as measured by modified transwell assays. Furthermore, the results from a bioluminescence tumor/metastasis model showed that the overexpression of MMP-1 significantly induces prostate tumor growth and the incidence of lung metastasis. We observed that this increase in tumor growth correlates with an increase in tumor angiogenesis. In addition, we assessed the importance of MMP-1 expression in cell invasion and migration by inhibiting MMP-1 activity with specific inhibitor and antibodies. Blockade of MMP-1 activity inhibited prostate cancer cell migration and invasion in vitro. Treatment of mice with an MMP-1 specific inhibitor significantly decreased prostate tumor growth and incidence of lung metastasis in vivo. Collectively, our findings suggest that MMP-1 plays an important role in prostate cancer progression during the invasive and metastatic stages of the disease.
\end{abstract}

\section{Introduction}

Approximately 27,050 prostate cancer-related deaths are predicted for this year alone in the United States. Despite recent improvements in diagnostic and therapeutic techniques, the survival rate of prostate cancer patients remains poor due to post-treatment recurrence of disease $(1,2)$. The lack of effective therapies for advanced prostate cancer is related to a

Correspondence to: Dr J.S. Rao, Department of Cancer Biology and Pharmacology, University of Illinois College of Medicine, Box 1649, Peoria, IL 61656, USA

E-mail: jsrao@uic.edu

Key words: MMP-1, invasion, angiogenesis, metastasis, prostate cancer large extent to a poor understanding of the molecular changes underlying the progression of the disease toward invasion and metastasis (3). Thus, the identification of new predictive biomarkers, which can serve as targets for more effective therapeutic and chemopreventative interventions, will improve clinical management of prostate cancer.

Tumor metastasis occurs by a series of steps including vessel formation, cell attachment, invasion and cell migration. As such, the regulation of metastasis is extremely complicated $(4,5)$. Degradation of basement membranes and the stromal extracellular matrix are crucial steps for tumor invasion and metastasis. Matrix metalloproteinases (MMPs) comprise a family of human zinc-dependent endopeptidases responsible for degradation of the extracellular matrix $(6,7)$. Among the MMPs, the collagenase MMP-1 has been identified as one of the most highly upregulated proteins in a variety of cancers, including colorectal, esophageal, pancreatic, gastric, breast, and malignant melanoma (8-13). Furthermore, elevated MMP-1 expression in atypical ductal hyperplastic tissues may serve as a marker for predicting which patients will develop invasive breast cancer (14). However, there are no reports yet on its relationship with prostate cancer metastasis.

The present study was designed to examine the effect of MMP-1 overexpression on cell migration, invasion, angiogenesis and metastasis. We found that overexpressing MMP-1 in human prostate cancer cells promotes primary tumor growth and leads to increased lung metastases. Furthermore, blocking MMP-1 expression inhibits tumor cell migration, invasion, angiogenesis and metastasis. These findings indicate that MMP-1 is a novel target for therapeutic applications in prostate cancer.

\section{Materials and methods}

Cell culture and transfection conditions. The prostate cancer cell lines RWPE2, LNCaP, DU145 and PC3 were obtained from the American Type Culture Collection (Manassas, VA). LNCaP cells were cultured in RPMI medium supplemented with $2 \mathrm{mM}$ L-glutamine, $1.5 \mathrm{~g} / \mathrm{l}$ sodium bicarbonate, $4.5 \mathrm{~g} / \mathrm{l}$ glucose, $10 \mathrm{mM}$ HEPES, and $1.0 \mathrm{mM}$ sodium pyruvate (Invitrogen, Carlsbad, CA). PC3 and DU145 cells were cultured in Dulbecco's modified Eagle's medium (DMEM)/ F12K (1:1). Both media contained 10\% fetal bovine serum (Gibco BRL, Lewisville, TX) and 5\% penicillin/streptomycin. RWPE2 cells were grown in K-SFM containing $50 \mu \mathrm{g} / \mathrm{ml}$ 
bovine pituitary extract, $5 \mathrm{ng} / \mathrm{ml}$ epidermal growth factor, and $1 \%$ penicillin/streptomycin solution. All cells were cultured in a $37^{\circ} \mathrm{C}$ incubator with a $5 \% \mathrm{CO}_{2}$ humidified atmosphere. The MMP-1 expression vector (pCMV-neo/MMP-1) was prepared by inserting full-length human MMP-1 cDNA into a pCMV-neo vector (OriGene, Rockville, MD). The MMP-1 expression vector and empty vector-transfected cells were selected by growth in the presence of G418 $(500 \mu \mathrm{g} / \mathrm{ml})$, and then analyzed for MMP-1 expression using RT-PCR and immunoblotting.

Collagen zymography. Confluent prostate cells were maintained in serum-free media for $24 \mathrm{~h}$. Equal amounts of conditioned media were loaded onto $10 \%$ polyacrylamide gels containing $1 \mathrm{mg} / \mathrm{ml}$ of collagen (Sigma, St. Louis, MO). After electrophoresis, the gels were washed and stained as described previously (15). The substrate-degrading enzyme MMP-1 was identified as clear bands in the blue background.

Invasion and cell migration assay. We used 6.5-mm diameter Transwell inserts (Costar, Cambridge, MA) with the $8-\mu \mathrm{m}$ pore membranes coated with Matrigel (Becton-Dickinson, Bedford, MA) to assess the invasive potentials of prostate cancer cells. Depletion of MMPs with antibodies and inhibitor was carried out as described previously (16). Cells were detached, washed twice in PBS and resuspended in serum-free DMEM. A total of $5 \times 10^{4}$ cells in $0.2 \mathrm{ml}$ were placed in each chamber as described (17). After a 24-h incubation period, the cells in the upper chamber that did not migrate were gently scraped away and adherent cells present on the lower surface of the insert were stained with Hema-3 and photographed. For assessing cell migration, the assay was carried out essentially as above, except that the cells were plated on top of uncoated transwell inserts and incubated for $8 \mathrm{~h}$.

In vitro angiogenesis assay. The endothelial tube-like formation assay was performed as described previously (6). Human dermal endothelial cells (HDECs) were seeded on 8 -well chamber slides at $4 \times 10^{4}$ cells per well. After cells adhered to the well $(12 \mathrm{~h})$, growth medium was replaced by conditioned medium. The conditioned media were collected from PC3 prostate cell cultures, which were pretreated with MMP-1 or VEGF neutralized antibodies at the indicated concentrations for $2 \mathrm{~h}$ at $37^{\circ} \mathrm{C}$ before being added to HDEC. After a 48-h culture in the treated media, dead floating cells were washed away. To assess tube formation, the attached living cells were stained with anti-vWF (Dako, Carpenteria, CA). We quantified tube formation by measuring the number of branch points and the total number of branches per point in triplicate wells.

Dorsal skin-fold chamber model. Tumor angiogenesis was quantified in vivo using the transparent dorsal skin-fold chamber model (18). Briefly, after total body anesthesia with ketamine $(50 \mathrm{mg} / \mathrm{kg})$ and xylazine $(10 \mathrm{mg} / \mathrm{kg})$, a dorsal air sac was made in the mouse by injecting $10 \mathrm{ml}$ of air. After dorsal skin-fold preparation, chambers were inoculated with $2 \times 10^{6}$ prostate cancer cells. Ten days later, the animals were anesthetized and sacrificed. The animals were carefully skinned around the implanted chambers, and the implanted chambers were removed from the subcutaneous air fascia. The skin fold covering the chambers was photographed under visible light. The number of blood vessels within the chamber in the area of the air sac fascia was counted, and their lengths were measured.

Reverse transcription-PCR analysis. Cellular RNA was isolated using the Qiagen RNeasy kit and $1 \mu \mathrm{g}$ of RNA was DNase-treated (10 U/ $\mu \mathrm{g}$ of RNA, $1 \mathrm{~h}$ ) and used as a template for the reverse transcription reaction (RT, $20 \mu \mathrm{l})$. RT reaction mix (Invitrogen) contained $1 \mu 1(10 \mathrm{pm})$ of primers. The resultant cDNA was then used in PCR reactions and analyzed by gel electrophoresis. The following primers were used: MMP-1sense, 5'-CGA CTC TAG AAA CAC AAG AGC AAG A-3', and MMP-1 antisense, 5'-AAG GTT AGC TTA CTG TCA CAC GCT T-3'; GAPDH-sense, 5'-CGG AGT CAA CGG ATT TGG TCG TAT-3', and GAPDH-antisense, 5'-AGC CTT CTC CAT GGT GGT GAA GAC-3'. The PCR conditions were as follows: $95^{\circ} \mathrm{C}$ for $5 \mathrm{~min}$, followed by 40 cycles at $95^{\circ} \mathrm{C}$ for $1 \mathrm{~min}, 58^{\circ} \mathrm{C}$ for $1 \mathrm{~min}$ and $72^{\circ} \mathrm{C}$ for $1 \mathrm{~min}$. The final extension was at $72^{\circ} \mathrm{C}$ for $5 \mathrm{~min}$.

Immunoblot analysis. Cells were lysed in RIPA buffer and proteins quantified using a BCA assay (Pierce, Rockford, IL). Equal amounts of proteins in the samples were separated on SDS-PAGE gels. Membranes were probed with antibodies against MMP-1 (Biomeda, Foster City, CA) and GAPDH (Abcam, Cambridge, MA). Horseradish peroxidase-conjugated secondary antibodies (Biomeda, Foster City, CA) were used for detection of immunoreactive proteins by chemiluminescence (Amersham Biosciences, Piscataway, NJ).

Orthotopic mouse prostate tumor/metastasis model. Athymic male nude mice (nu/nu; 6-8 weeks old) were obtained from Harlan Sprague-Dawley (Indianapolis, IN). Animal handling and experimental procedures were approved by the University of Illinois College of Medicine Institutional Animal Care and Use Committee. Orthotopic implantation was carried out as described previously with minor modifications (19). Briefly, after total body anesthesia with ketamine $(50 \mathrm{mg} / \mathrm{kg}$ ) and xylazine $(10 \mathrm{mg} / \mathrm{kg})$, a small incision was made to reveal the prostate gland. LNCaP cells stably expressing either empty vector or MMP-1 expression vector with luciferase reporter were injected into mouse prostate $\left(10^{6}\right.$ cells per mouse). Prostate tumor growth and subsequent metastasis to lungs were assessed weekly using the in vivo imaging system coupled to Living Image acquisition and analysis software (Xenogen, Alameda, CA) according to the manufacturer's instructions. For inhibitor studies, $10^{6}$ PC3-luc cells in PBS were injected into the mouse prostate. MMP-1 inhibitor (FN-439; Calbiochem, San Diego, CA) was injected intraperitoneally at a dose of $5 \mathrm{mg} / \mathrm{kg}$ twice a week for a maximum of 6 weeks. All mice were sacrificed at either 6 weeks postimplantation or when clinical conditions dictated euthanasia. We then excised, measured and weighed the prostate tumors. Part of the tumor was snap-frozen for RT-PCR analysis, and the remaining portion was placed in phosphate-buffered formalin and then ethanol before preparing paraffin sections for hematoxylin and eosin staining. 

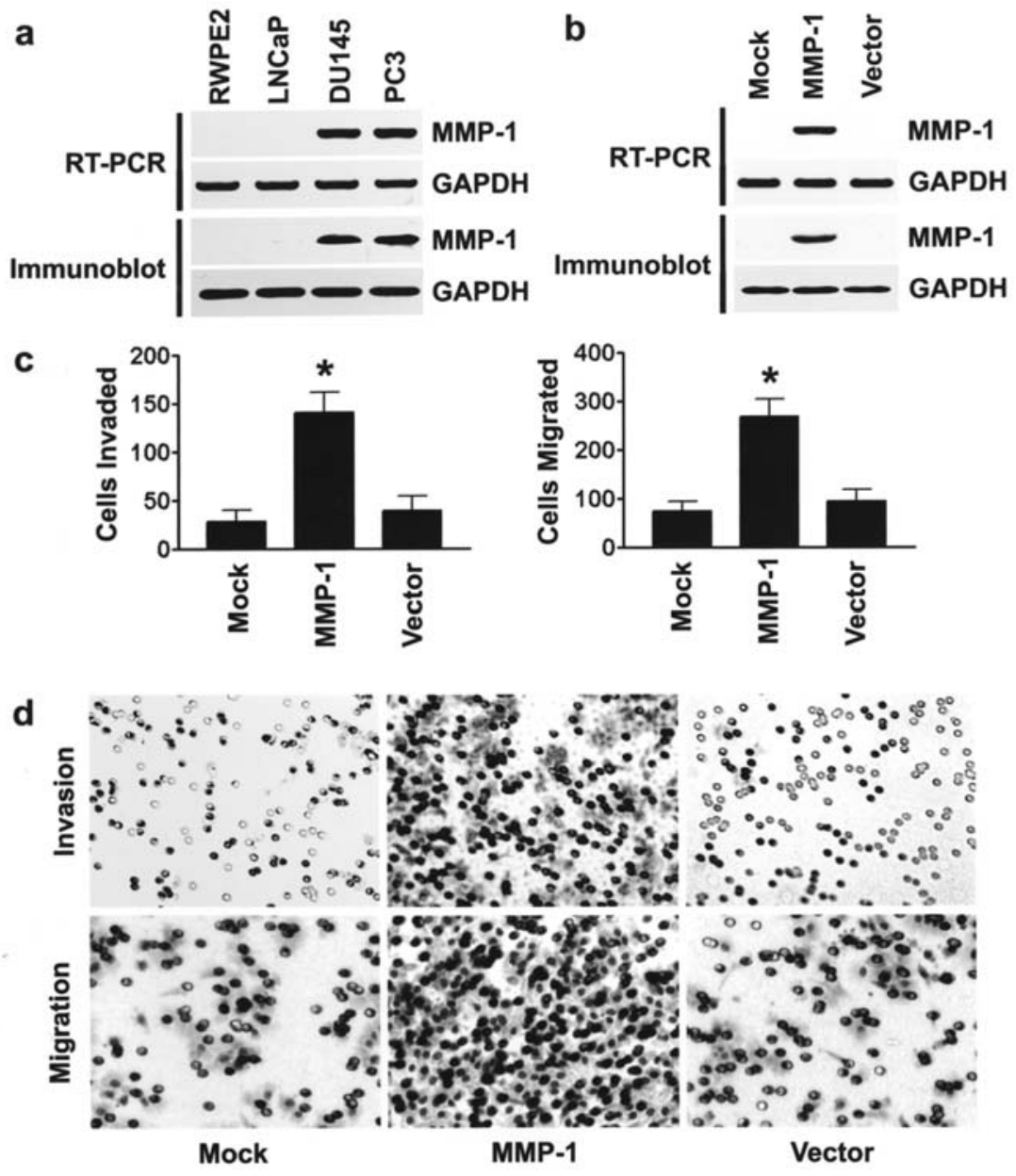

Figure 1. Overexpression of MMP-1 enhances invasiveness and cell migration of LNCaP prostate cancer cells. (a) mRNA (top) and protein (bottom) expression levels of MMP-1 in prostate cancer cell lines. GAPDH was used as a loading control for RNA and protein analyses. (b) RT-PCR (top) and immunoblot (bottom) analyses of LNCaP cells stably transfected with mock, MMP-1 expression vector or empty vector. (c) Comparison of the in vitro migration (right) and invasive (left) potentials of LNCaP cells stably transfected with mock, MMP-1 expression vector or empty vector. Columns, mean of three independent experiments $\left({ }^{*} \mathrm{p}<0.01\right)$; bars, SD. (d) Representative invasion and migration photographs from LNCaP cells stably transfected with mock, MMP-1 expression vector or empty vector as described in (c).

Histological analysis and immunohistologic staining. Tissues were fixed with formalin, embedded in paraffin, sectioned $(5 \mu \mathrm{m})$ and stained with hematoxylin and eosin. Immunostaining was performed according to standard techniques using a polyclonal antibody specific for human vWF (Dako). Visualization of antibody binding was performed by the second antibody tagged with HRP following reaction to DAB (Sigma). Microvessel density was determined as described previously (17).

Statistical analysis. Statistical comparisons were performed using ANOVA for analysis of significance between different values using GraphPad Prism software (San Diego, CA). Values are expressed as mean \pm SD from at least 3 separate experiments and differences were considered significant at a $\mathrm{p}<0.05$.

\section{Results}

Overexpression of MMP-1 enhances invasiveness and cell migration of prostate cancer cells. To study the possible role of MMP-1 expression in the invasion and migration of prostate cancer cells, we generated MMP-1-overexpressing cells. We initially examined MMP-1 mRNA and protein levels in the prostate cancer cell lines RWPE2, LNCaP, DU145 and PC3. Among the four cell lines, only DU145 and PC3 cells highly expressed MMP-1 at both the mRNA and protein levels (Fig. 1a). LNCaP, an MMP-1 null prostate cancer cell line that has a very low invasion index (19), was transfected with either an MMP-1 expression vector or an empty vector. We observed high expression of MMP-1 in MMP-1-transfected cells but not in parental (mock) or empty vector-transfected cells as detected by RT-PCR and immunoblotting (Fig. 1b). Differences in invasion between MMP-1-expressing cells and control LNCaP cells were evaluated using a Matrigel invasion assay. The results show that MMP-1 stably expressing LNCaP cells were significantly more invasive than the empty vector controls (Fig. 1c, left). In addition, the rate of cell migration between these cells was also compared using the two-chamber assay with uncoated inserts (instead of Matrigel-coated filters). Interestingly, LNCaP cells stably expressing MMP-1 showed increased migration when compared with the empty vector controls (Fig. 1c, right). Representative pictures are shown in Fig. 1d. 
a

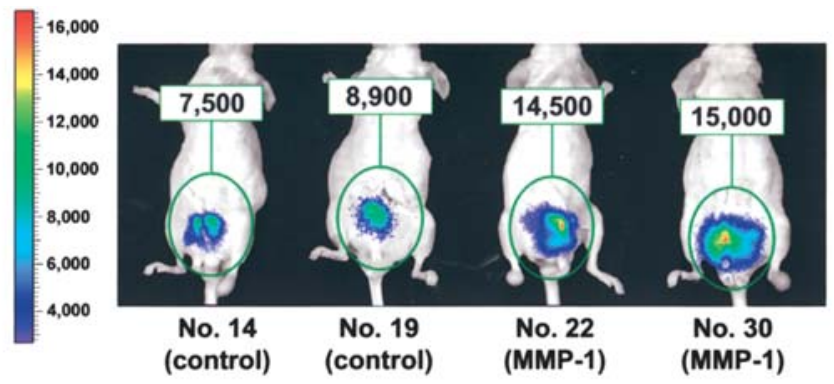

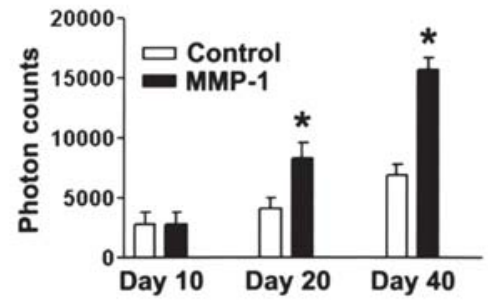

b
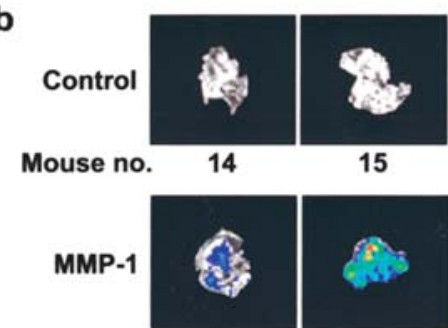

Mouse no.
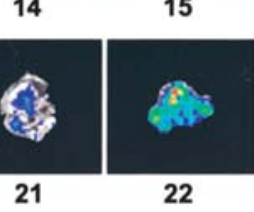

22

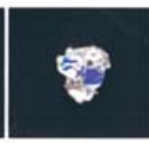

11

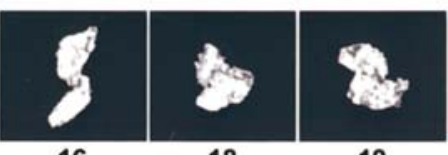

16

18

19

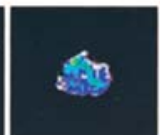

25

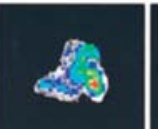

27

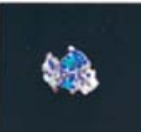

29

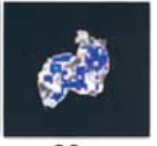

30

C
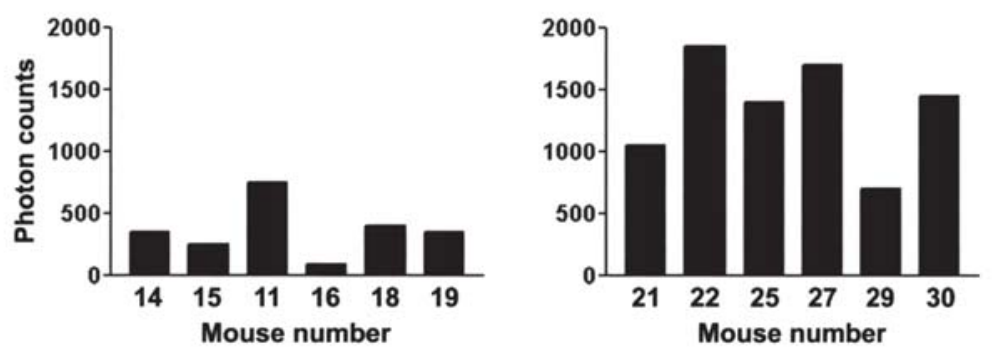

d

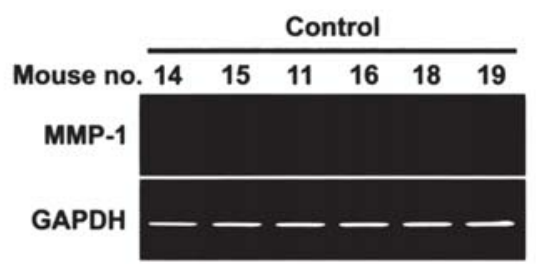

MMP-1

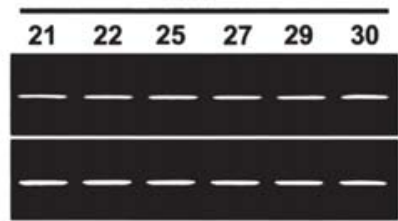

Figure 2. MMP-1 overexpression promotes prostate tumor growth and metastasis in nude mice. (a) LNCaP cells stably expressing luciferase reporter with either empty vector or MMP-1 expression vector were injected into mouse prostate, and luciferase activity was recorded for each mouse. Representative mice images are shown (left). The colour bar represents luciferase intensity. Numbers relating to the green ovals represent photon counts in the prostate. Photon counts of orthotopic prostate tumors on days 10, 20 and 40 (right). Significant differences from control are indicated by an asterisk $(*)$ (p<0.01). (b) Luciferase activity in the lung, representative of lung metastasis, was recorded for each mouse. Lung images from different mice are shown. (c) Quantification of luciferase activities from either each control mouse (left) or each MMP-1 expressing mouse (right). (d) RNA samples extracted from prostate tumors (6 animals/group) were analyzed using RT-PCR for MMP-1 expression levels. GAPDH mRNA was amplified as a loading control and expression standard.

Furthermore, to extend these findings to an additional prostate cancer cell line, RWPE2 cells were stably transfected with the MMP-1 expression vector and tested for invasion and migration. Similar to the results described above, RWPE2 cells stably expressing MMP-1 exhibited increased invasion and migration when compared with RWPE2 cells that did not express MMP-1 (data not shown).

MMP-1 overexpression promotes tumor growth and metastasis in nude mice. We tested whether the invasive phenotype conferred by MMP-1 in LNCaP cells under in vitro conditions could be extended to an in vivo tumor model system. To produce orthotopic tumors in nude mice, LNCaP cells stably expressing either empty vector (control) or MMP-1 expression vector with luciferase reporter were injected into the prostate of immunodeficient mice. Tumor progression was monitored in mice using the Xenogen in vivo imaging system. We obtained photon counts from the tumor region on days 10, 20 and 40 (Fig. 2a, right). The mice injected with LNCaP cells stably transfected with MMP-1 developed larger prostate tumors by day 40 when compared with the mice inoculated with cells transfected with the empty vector (Fig. 2a, left). The presence of significant growth differences in prostate tumors between control and mice injected with MMP-1-overexpressing cells were confirmed by autopsy after imaging (data not shown). To determine the effect of MMP-1 on tumor metastasis, we dissected the lungs from each mouse and photon counts were recorded. The lungs derived from control mice injected with empty vectortransfected cells had low photon counts. In contrast, we observed a marked induction (3.7-fold) in the incidence of lung metastasis when primary tumor cells stably transfected with MMP-1 were seeded in host mice (Fig. 2b and c). RT-PCR analysis confirmed expression of MMP-1 in prostate tumors developed from the LNCaP cells stably expressing MMP-1 (Fig. 2d). These results clearly show that expression 
a

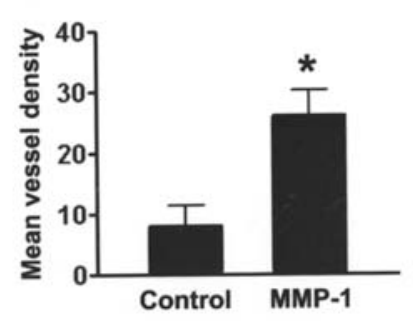

b

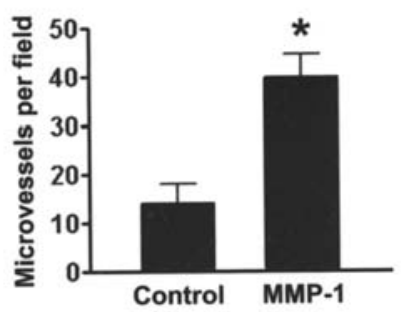

C

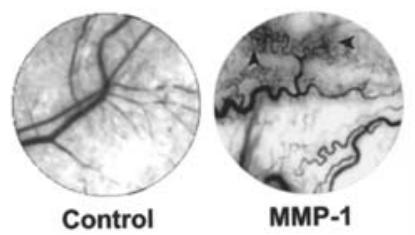

Figure 3. MMP-1 overexpression induces tumor angiogenesis. (a) Cells positive for vWF were counted in five high-power fields in tumor sections with indicated groups ( $\mathrm{n}=6$ tumors from each group). Tissue sections $(5 \mu \mathrm{m})$ were prepared and stained with control IgG or anti-vWF. Significant differences from control are indicated by an asterisk $(*)(\mathrm{p}<0.01)$. (b) Tumor angiogenesis was monitored in the dorsal skin-fold chamber of an immunodeficient mouse. Microvessels were counted under a microscope in five random fields. The results represent mean \pm SD from six implants per group ( ${ }^{*} \mathrm{p}<0.01$ ). (c) Representative photographs show changes in microvessel density of mouse skin-folds in response to the secretions of LNCaP cells stably expressing empty vector or MMP-1 expression vector as described in (c). Arrowheads indicate newly formed blood vessels in the skin-folds.

of MMP-1 in LNCaP prostate cancer cells significantly enhances both primary tumor growth and the in vivo incidence of lung metastasis.

Effect of MMP-1 overexpression on angiogenesis in vivo. We investigated whether the accelerated tumor growth and metastasis that we observed could be due to tumor angiogenesis stimulated by MMP-1. Immunohistochemical staining for endothelial cells revealed much higher vWF-positive vascular structures within the prostate tumors developed from MMP-1overexpressing cells than the empty vector controls (Fig. 3a). Next, we used a dorsal skin-fold chamber model to further characterize the effects of MMP-1 overexpression on tumor angiogenesis in vivo. Although the implantation of a chamber containing cells transfected with empty vector did not promote tiny new vascular sprouts and branches similar to those that grew from pre-existing microvessels, there was a significant increase in angiogenic response in the skin-folds of mice implanted with chambers containing MMP-1-overexpressing cells (Fig. 3b). Representative pictures are shown in Fig. 3c. These results suggest that enhanced MMP-1 expression increased angiogenic activity in prostate tumors.

MMP-1 depletion reduces invasion and migration of prostate cancer cells. To further confirm the role of MMP-1 overexpression on the invasive properties of prostate cancer cells, we tested the effect of selective pharmacologic inhibition of MMP-1 on cell invasion and migration. We carried out inhibition studies using the well-characterized MMP-1 inhibitor FN-439 $(16,20,21)$. Our results showed the MMP-1 inhibitor induced a dose-dependent regulation on invasion; cells treated with the MMP-1 inhibitor at low doses of 1-100 nM showed no significant differences in invasion, whereas a higher dose of MMP-1 inhibitor $(10 \mu \mathrm{M})$ significantly diminished the invasive potential of metastatic DU145 and PC3 cells (Fig. 4a). We also assessed in vitro migration in response to the MMP-1 inhibitor using a Transwell migration assay. MMP-1 inhibitor suppressed the migration of DU145 and PC3 cells through uncoated, porous filters in a dose-dependent manner, with no significant effect at a concentration of 1-100 $\mathrm{nM}$ and a maximal effect at $10 \mu \mathrm{M}$ (Fig. 4b). Collagen zymography showed that $10 \mu \mathrm{M}$ of MMP-1 inhibitor treatment significantly reduced MMP-1 activity in DU145 and PC3 cells (Fig. 4c). In addition, we immunodepleted individual MMPs from DU145 and PC3 conditioned medium with the corresponding anti-human MMP antibodies. As shown in Fig. 4d, immunodepletion of MMP-1 from DU145 and PC3 conditioned medium caused a significant drop in cell invasion (left) and migration (right). Treatment of the DU145 and PC3 conditioned medium with antibodies directed against MMP-2 resulted in a slight drop in cell invasion and migration. However, simultaneous immunodepletion of MMP-1 and MMP-2 caused the same drop in invasion and migration as immunodepletion of MMP-1 alone. No effects were observed upon treatment with anti-MMP-3, -7 or -9 antibodies. Together, these data demonstrate that MMP-1 is capable of inducing invasion and migration in prostate cancer cells.

$M M P-1$ inhibitor suppresses in vitro and in vivo angiogenesis of prostate cancer cells. Because tumor angiogenesis involves multiple factors, including endothelial cells (ECs) and tumor cells, we first tested whether PC3 cells exerted a pro-angiogenic effect on neighboring ECs through MMP-1 and other angiogenic proteins. We used the EC tube-like network formation assay, an in vitro assay that correlates well with in vivo angiogenic activity $(22,23)$. EC networks increased 5.1-fold following exposure to PC3 conditioned media compared to basal media. Further, blocking antibodies to both MMP-1 and VEGF were able to abolish the angiogenic effects of the PC3 conditioned media on EC network formation (Fig. 5a). However, because MMP-1 depletion alone resulted in a strong inhibition of network formation, we could not demonstrate an additive or synergistic effect of MMP-1 and VEGF inhibition. These data demonstrate the ability of MMP-1 to induce EC network formation. Notably, we found that conditioned media from PC3 cells treated with $10 \mu \mathrm{M}$ of MMP-1 inhibitor suppressed tube formation (Fig. 5b). Representative pictures are shown in Fig. 5c. We next used a dorsal skin-fold chamber model to further characterize the effects of MMP-1 inhibitor on tumor angiogenesis in vivo. Control experiments containing untreated PC3 cells did not interfere with neovascularization as shown by the formation of new vessels from the preexisting vasculature. In contrast, we observed significantly less angiogenic response in the skin-folds of mice implanted 
a
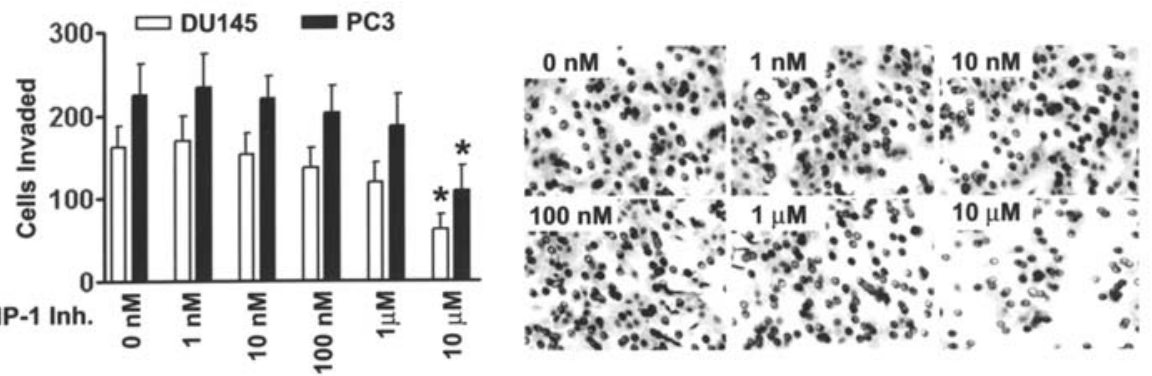

b
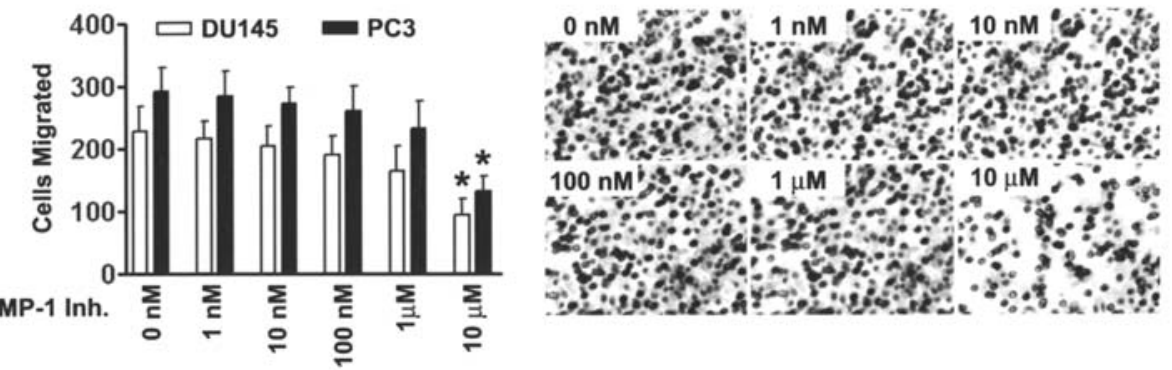

C

DU145
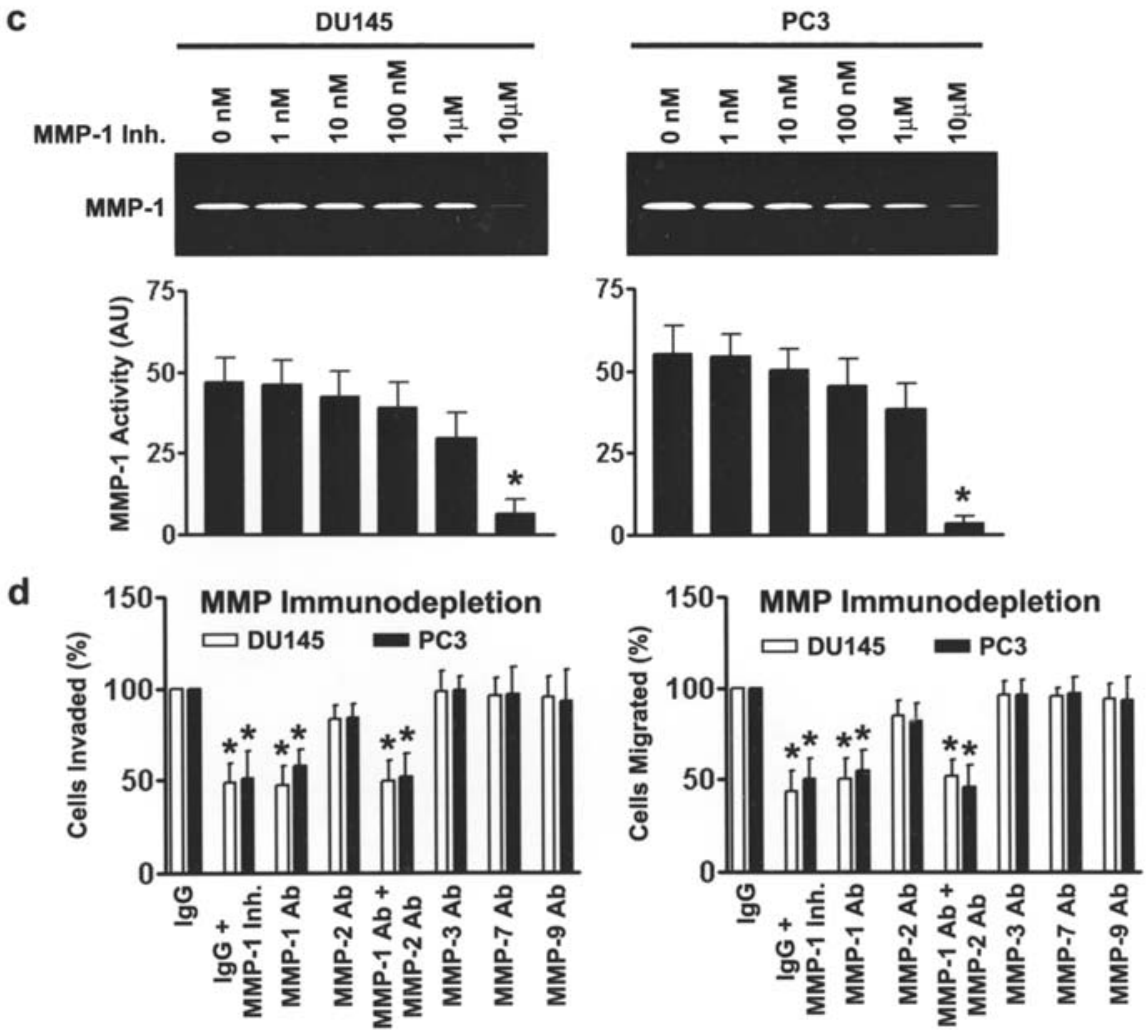

Figure 4. MMP-1 depletion reduces invasion and migration of prostate cancer cells. (a) The invasive potential of DU145 and PC3 cells with the indicated treatments was examined as described in Fig. 1c. Representative invasion photographs are shown (right). (b) Migration of DU145 and PC3 cells with the indicated treatments was examined as described in Fig. 1c. Representative migration photographs are shown (right). (c) MMP-1 activity in DU145 and PC3 cells with the indicated treatments was accessed by collagen zymography. MMP-1 activity was detected as clear lysis bands after Amido Black staining and subsequent destaining with methanol/acetic acid. Bar diagram showing densitometry quantified mean values from three separate experiments ("p $<0.01)$. (d) Invasion (left) and migration (right) of DU145 and PC3 cells were inhibited by immunodepletion of MMP-1. DU145 and PC3 cells in serum-free DMEM were incubated with the indicated individual MMPs or IgG control $\left(30 \mathrm{~min}, 22^{\circ} \mathrm{C}\right)$ and then used for transwell assays as described in Materials and methods. Significant differences from control are indicated by an asterisk $(*)(\mathrm{p}<0.01)$.

with chambers containing MMP-1 inhibitor-treated PC3 cells (Fig. 5d). These data suggest that MMP-1 inhibitor suppressed angiogenesis both in vitro and in vivo.

Pharmacological inhibition of MMP-1 reduces tumor growth and metastasis in nude mice. Having shown that the MMP-1 inhibitor affects prostate cancer cell invasion and angiogenesis, we investigated the effects of pharmacologic blockade of MMP-1 on the growth of PC3 orthotopic tumors in nude mice. We injected $1 \times 10^{6} \mathrm{PC} 3$ cells stably expressing luciferase into the prostate of immunodeficient mice. Starting on day 6 after injection, mice were treated with either vehicle control or MMP-1 inhibitor (5 mg/kg intraperitoneally twice a week for a maximum of 6 weeks). Tumor progression was monitored 


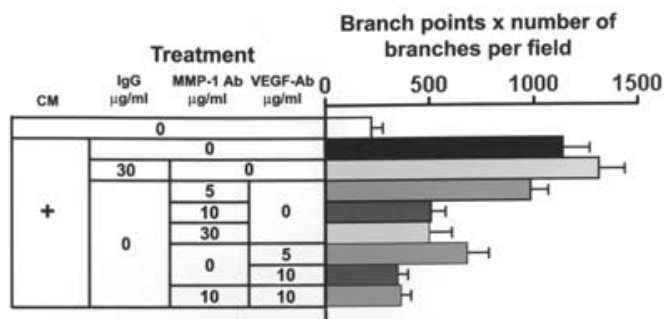

b

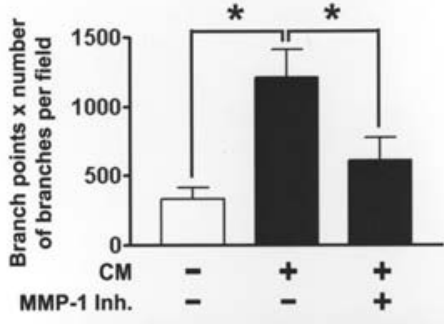

C

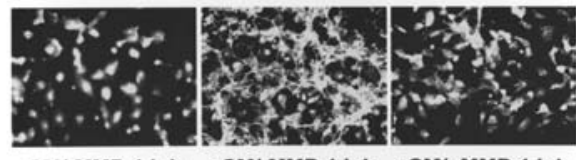

-CM/-MMP-1 Inh. +CM/-MMP-1 Inh. +CM/+MMP-1 Inh.

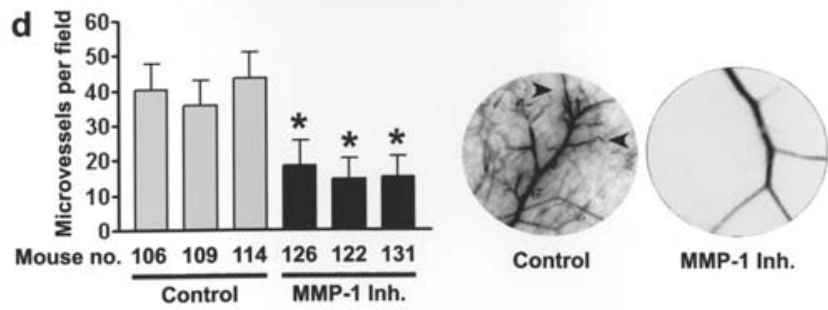

Figure 5. MMP-1 inhibitor suppresses in vitro and in vivo angiogenesis of prostate cancer cells. (a) The PC3-derived conditioned media significantly increased EC tube-like network formation in culture, an effect nearly completely blocked by neutralizing antibodies to either MMP-1 or VEGF. (b) Angiogenic activity in media treated with MMP-1 inhibitor or control cell culture using the EC tube-like formation assay. Columns, mean of three independent experiments $\left({ }^{*} \mathrm{p}<0.01\right)$; bars, SD. (c) Representative angiogenic activity photographs in media treated with MMP-1 inhibitor or control cell culture as described in (b). (d) Angiogenesis was monitored in dorsal skinfold chamber after implantation of PC3 cells treated or untreated with MMP-1 inhibitor in nude mice. The results represent mean \pm SD from individual mice tested in each group are shown (left) $\left({ }^{*} \mathrm{p}<0.01\right)$. Representative photographs showing changes in microvessel density of mouse skin-folds are shown (right).

in mice using the Xenogen in vivo imaging system. We obtained photon counts from the tumor region on days 10, 20 and 40 (Fig. 6a, right). The tumors rapidly grew in control mice, resulting high photon counts in all control mice by day 40 . In contrast, mice receiving the MMP-1 inhibitor developed prostate tumors of significantly smaller volume by day 40 (Fig. 6a, left). We also assessed the efficacy of drug treatments as interventions during the natural formation of lung metastasis from prostate tumors. Although the lungs derived from control mice had high photon counts, there was a significant decrease in lung metastases in the MMP-1 inhibitor-treated mice (Fig. 6b). RT-PCR analysis confirmed MMP-1 inhibition in prostate tumors developed from mice treated with the MMP-1 inhibitor (Fig. 6c). Immunohistochemical staining for endothelial cells revealed prominent vWF-positive vascular structures within the tumor mass of the control groups. In contrast, significantly less vascularity was present in tumors developed from mice treated with the a
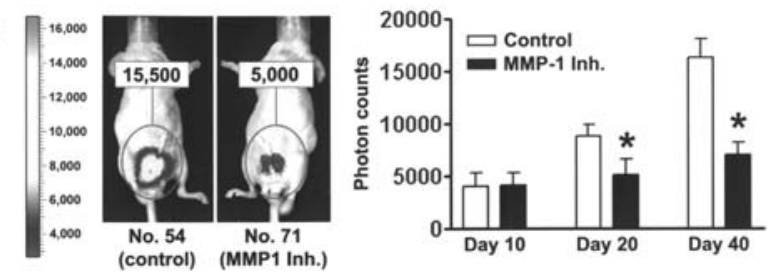

b
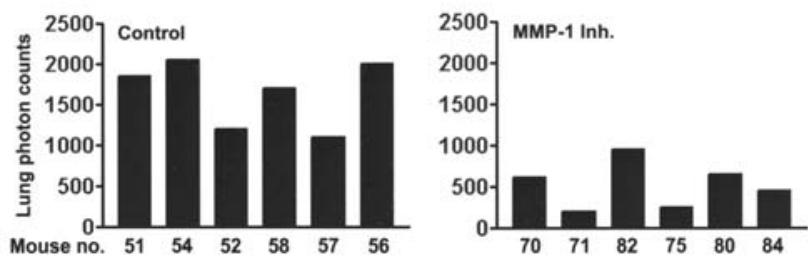

c

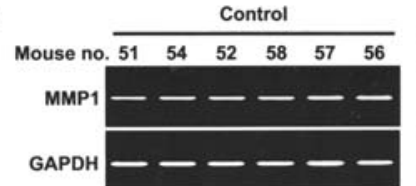

d

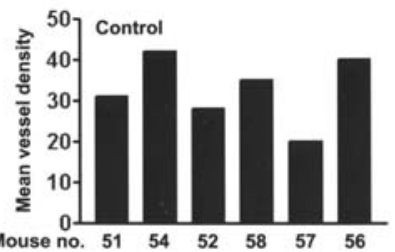

gure 6. Pharmacological inhibition of MMP-1 reduces tumor growth and metastasis in nude mice. (a) PC3 cells stably expressing luciferase vector (PC3-luc) were injected into mouse prostate and the tumors allowed to grow for 7 days before initiation of treatment with saline or MMP-1 inhibitor. Representative images are shown (left). The color bar represents luciferase intensity. Numbers relating to the green ovals represent photon counts in the prostate. Photon counts of orthotopic prostate tumors on days 10, 20 and 40 (right). Significant differences from control are indicated by an asterisk $(*)$ $(\mathrm{p}<0.01)$. (b) Quantification of luciferase activities from either each control mouse (left) or each MMP-1 inhibitor-treated mouse (right). (c) RNA samples extracted from prostate tumors (6 animals/group) were analyzed using RTPCR for MMP-1 expression levels. GAPDH mRNA was amplified as a loading control and expression standard. (d) Cells positive for vWF were counted in five high-power fields in tumor sections with indicated groups. The results represent mean \pm SD from individual mice tested in each group are shown.

MMP-1 inhibitor (Fig. 6d). These results suggest that MMP-1 can promote tumor growth, angiogenesis and metastasis, but significant inhibition of these processes can be achieved by inhibition of MMP-1.

\section{Discussion}

We investigated the potential implication of the MMP-1 in prostate cancer progression. This study provides the first direct evidence for a pivotal role of MMP-1 in primary prostate cancer growth, leading to metastatic dissemination. Several lines of evidence support these conclusions. First, MMP-1 is only expressed in the highly invasive prostate cancer cell lines DU145 and PC3. Second, the overexpression of MMP-1 in LNCaP prostate cancer cells, which have a low spontaneous metastasis rate, can promote in vitro tumor cell invasion as well as significantly enhance in vivo primary tumor growth and spontaneous metastatic potential. Third, overexpression of MMP-1 in LNCaP cells results in an enhancement of 
tumor angiogenesis. Fourth, blocking MMP-1 expression in PC3 prostate cancer cells significantly inhibited prostate tumor growth and the incidence of lung metastasis.

The regulation of cell invasion and migration is critical to the progression of cancer. Our studies show that MMP-1 overexpression enhances both the invasion and migration of human prostate cancer cells in vitro (Fig. 1). In addition, we show that overexpression of MMP-1 is sufficient to promote growth and invasion of prostate cancer cells in an orthotopic tumor model (Fig. 2). This orthotopic prostate tumor model seems clinically relevant because its tumor progressioncontaining metastasis mimics the clinical scenario. Our findings are in agreement with previous observations in colorectal carcinoma and breast cancer where overexpression of MMP-1 had a suggested potential role in cancer promotion $(11,14)$.

Another interesting finding of our study was that overexpression of MMP-1 significantly induced tumor angiogenesis in vivo (Fig. 3). Parts of the biological characteristics of tumor cells are closely associated with those of microvascular endothelial cells in tumor tissues (24). Endothelial cell secretion of MMP-1 has been shown to assist angiogenesis in vivo (25), and it follows that tumor cell production of MMP-1 should facilitate blood vessel growth in the tumor microenvironment. In addition, several investigators have reported that MMP-1 could stimulate endothelial cell growth $(20,26)$. Furthermore, Goerge et al (20) reported that tumorderived MMP-1 is crucial for angiogenesis. Our results provide support to these findings.

There is now substantial evidence that the family of MMPs is important for various aspects of prostate tumorigenesis. Functionally, inhibitors of metalloproteinase activity attenuate the growth and invasion of tumors cells implanted into the flank $(27)$ or prostate of rats $(28,29)$. Our study also examined the specific effect of pharmacologic inhibition of MMP-1 on prostate tumor cell invasion, angiogenesis and metastasis (Figs. 4, 5 and 6). Although it could be speculated that tetrapeptidyl hydroxamic acid (FN-439) is not specific for MMP-1, it was shown previously that MMP inhibitor FN-439 targets MMP-1 but not MMP-2 or MMP-3 (20). Consistent with this notion, we found that FN-439 significantly reduced MMP-1 activity (Fig. 4c), but had no effect on MMP-9 or MMP-2 in DU145 and PC3 cells (data not shown). In addition, DU145 and PC3 cells had undetectable levels of MMP-3, MMP-7 and MMP-8 (data not shown). Taken together, these data strengthen our notion that MMP-1 plays an important role during the progression of human prostate cancer.

In conclusion, our studies have revealed the critical role of MMP-1 in invasion and tumor growth in the metastatic process. These findings provide new and important information on the progression of prostate cancer. The finding that MMP-1 influences metastatic potential, as shown in this study, raises the possibility that MMP-1 could be used as a molecular target in the anti-metastatic therapy of patients with prostate cancer.

\section{Acknowledgements}

We thank Shellee Abraham for preparing the manuscript, and Diana Meister and Sushma Jasti for manuscript review. This research was supported by National Cancer Institute Grant CA 75557, CA 92393, CA 95058, CA 116708 and N.I.N.D.S.
NS47699, NS57529 and Caterpillar, Inc., OSF Saint Francis, Inc., Peoria, IL (to J.S.R.).

\section{References}

1. Jemal A, Siegel R, Ward E, Murray T, Xu J and Thun MJ: Cancer statistics, 2007. CA Cancer J Clin 57: 43-66, 2007.

2. Klein EA and Thompson IM: Update on chemoprevention of prostate cancer. Curr Opin Urol 14: 143-149, 2004.

3. Logothetis CJ and Lin SH: Osteoblasts in prostate cancer metastasis to bone. Nat Rev Cancer 5: 21-28, 2005.

4. Chambers AF, Groom AC and MacDonald IC: Dissemination and growth of cancer cells in metastatic sites. Nat Rev Cancer 2: 563-572, 2002.

5. Fidler IJ: The pathogenesis of cancer metastasis: the 'seed and soil' hypothesis revisited. Nat Rev Cancer 3: 453-458, 2003.

6. Lakka SS, Gondi CS and Rao JS: Proteases and glioma angiogenesis. Brain Pathol 15: 327-341, 2005.

7. Rao JS: Molecular mechanisms of glioma invasiveness: the role of proteases. Nat Rev Cancer 3: 489-501, 2003.

8. Airola K, Karonen T, Vaalamo M, Lehti K, Lohi J, Kariniemi AL, Keski-Oja J and Saarialho-Kere UK: Expression of collagenases-1 and -3 and their inhibitors TIMP- 1 and -3 correlates with the level of invasion in malignant melanomas. $\mathrm{Br} \mathrm{J}$ Cancer 80: 733-743, 1999.

9. Inoue T, Yashiro M, Nishimura S, Maeda K, Sawada T, Ogawa Y, Sowa M and Chung KH: Matrix metalloproteinase-1 expression is a prognostic factor for patients with advanced gastric cancer. Int J Mol Med 4: 73-77, 1999.

10. Ito T, Ito M, Shiozawa J, Naito S, Kanematsu $\mathrm{T}$ and Sekine I: Expression of the MMP-1 in human pancreatic carcinoma: relationship with prognostic factor. Mod Pathol 12: 669-674, 1999.

11. Murray GI, Duncan ME, O'Neil P, Melvin WT and Fothergill JE: Matrix metalloproteinase-1 is associated with poor prognosis in colorectal cancer. Nat Med 2: 461-462, 1996.

12. Murray GI, Duncan ME, O'Neil P, McKay JA, Melvin WT and Fothergill JE: Matrix metalloproteinase-1 is associated with poor prognosis in oesophageal cancer. J Pathol 185: 256-261, 1998.

13. Nakopoulou L, Giannopoulou I, Gakiopoulou H, Liapis H, Tzonou A and Davaris PS: Matrix metalloproteinase- 1 and -3 in breast cancer: correlation with progesterone receptors and other clinicopathologic features. Hum Pathol 30: 436-442, 1999.

14. Poola I, DeWitty RL, Marshalleck JJ, Bhatnagar R, Abraham J and Leffall LD: Identification of MMP-1 as a putative breast cancer predictive marker by global gene expression analysis. Nat Med 11: 481-483, 2005.

15. Gogly B, Groult N, Hornebeck W, Godeau G and Pellat B: Collagen zymography as a sensitive and specific technique for the determination of subpicogram levels of interstitial collagenase. Anal Biochem 255: 211-216, 1998.

16. Boire A, Covic L, Agarwal A, Jacques S, Sherifi S and Kuliopulos A: PAR1 is a matrix metalloprotease-1 receptor that promotes invasion and tumorigenesis of breast cancer cells. Cell 120: 303-313, 2005 .

17. Pulukuri SM and Rao JS: Small interfering RNA directed reversal of urokinase plasminogen activator demethylation inhibits prostate tumor growth and metastasis. Cancer Res 67: 6637-6646, 2007.

18. Leunig M, Yuan F, Menger MD, Boucher Y, Goetz AE, Messmer K and Jain RK: Angiogenesis, microvascular architecture, microhemodynamics, and interstitial fluid pressure during early growth of human adenocarcinoma LS174T in SCID mice. Cancer Res 52: 6553-6560, 1992.

19. Pulukuri SM, Gondi CS, Lakka SS, Jutla A, Estes N, Gujrati M and Rao JS: RNA interference-directed knockdown of urokinase plasminogen activator and urokinase plasminogen activator receptor inhibits prostate cancer cell invasion, survival, and tumorigenicity in vivo. J Biol Chem 280: 36529-36540, 2005.

20. Goerge T, Barg A, Schnaeker EM, Poppelmann B, Shpacovitch V, Rattenholl A, Maaser C, Luger TA, Steinhoff $M$ and Schneider SW: Tumor-derived matrix metalloproteinase-1 targets endothelial proteinase-activated receptor 1 promoting endothelial cell activation. Cancer Res 66: 7766-7774, 2006.

21. Odake S, Morita Y, Morikawa T, Yoshida N, Hori H and Nagai Y: Inhibition of matrix metalloproteinases by peptidyl hydroxamic acids. Biochem Biophys Res Commun 199: 1442-1446, 1994. 
22. Gondi CS, Lakka SS, Yanamandra N, Olivero WC, Dinh DH, Gujrati M, Tung CH, Weissleder R and Rao JS: Adenovirusmediated expression of antisense urokinase plasminogen activator receptor and antisense cathepsin B inhibits tumor growth, invasion, and angiogenesis in gliomas. Cancer Res 64: 4069-4077, 2004.

23. Lakka SS, Gondi CS, Dinh DH, Olivero WC, Gujrati M, Rao VH, Sioka C and Rao JS: Specific interference of uPAR and MMP-9 gene expression induced by double-stranded RNA results in decreased invasion, tumor growth and angiogenesis in gliomas. J Biol Chem 280: 21882-21892, 2005.

24. Streubel B, Chott A, Huber D, Exner M, Jager U, Wagner O and Schwarzinger I: Lymphoma-specific genetic aberrations in microvascular endothelial cells in B-cell lymphomas. N Engl J Med 351: 250-259, 2004.

25. Karelina TV, Goldberg GI and Eisen AZ: Matrix metalloproteinases in blood vessel development in human fetal skin and in cutaneous tumors. J Invest Dermatol 105: 411-417, 1995.

26. Masckauchan TN, Agalliu D, Vorontchikhina M, Ahn A, Parmalee NL, Li CM, Khoo A, Tycko B, Brown AM and Kitajewski J: Wnt5a signaling induces proliferation and survival of endothelial cells in vitro and expression of MMP-1 and Tie-2. Mol Biol Cell 17: 5163-5172, 2006.
27. Qu XJ, Yuan YX, Tian ZG, Xu WF, Chen MH, Cui SX, Guo Q, Gai R, Makuuchi M, Nakata M and Tang W: Using caffeoyl pyrrolidine derivative LY52, a potential inhibitor of matrix metalloproteinase-2, to suppress tumor invasion and metastasis. Int J Mol Med 18: 609-614, 2006.

28. Jung K, Krell HW, Ortel B, Hasan T, Romer A, Schnorr D, Loening SA and Lein M: Plasma matrix metalloproteinase 9 as biomarker of prostate cancer progression in Dunning (Copenhagen) rats. Prostate 54: 206-211, 2003.

29. Lein M, Jung K, Ortel B, Stephan C, Rothaug W, Juchem R, Johannsen M, Deger S, Schnorr D, Loening S and Krell HW: The new synthetic matrix metalloproteinase inhibitor (Roche 28-2653) reduces tumor growth and prolongs survival in a prostate cancer standard rat model. Oncogene 21: 2089-2096, 2002. 